International Journal of Pure and Applied Mathematics

Volume 85 No. 4 2013, 719-728

ISSN: 1311-8080 (printed version); ISSN: 1314-3395 (on-line version)

url: http://www.ijpam.eu

doi: http://dx.doi.org/10.12732/ijpam.v85i4.8

\title{
ANALOGOUS TO RAMANUJAN'S REMARKABLE PRODUCT OF THETA FUNCTION OF DEGREE 5 AND THEIR EXPLICIT EVALUATION
}

\author{
B.N. Dharmendra ${ }^{1}$, M.R. Rajesh Kanna ${ }^{2}$, H.L. Parashivamurthy ${ }^{3}$ \\ ${ }^{1,2}$ Post Graduate Department of Mathematics \\ Maharani's Science College for Women \\ J.L.B. Road, Mysore, 570 001, INDIA \\ ${ }^{3}$ Department of Mathematics \\ BGS Institute Of Technology \\ B.G. Nagar, Bellur, Nagamangala Thalque \\ Mandya District, 571448, INDIA
}

Abstract: In this paper, We study the Analogous of Ramanujan's Remarkable product of theta-function of degree 5 of and their explicit values.

AMS Subject Classification: 33D10, 40A15, 11A55, 30B70

Key Words: continued fraction, theta functions

\section{Introduction}

In Chapter 16 of his second notebook [2], Ramanujan develops the theory of theta-function and is defined by

$$
\begin{aligned}
f(a, b):=\sum_{n=-\infty}^{\infty} a^{\frac{n(n+1)}{2}} b^{\frac{n(n-1)}{2}}, \quad|a b| & 1, \\
& =(-a ; a b)_{\infty}(-b ; a b)_{\infty}(a b ; a b)_{\infty},
\end{aligned}
$$

where $(a ; q)_{0}=1$ and $(a ; q)_{\infty}=(1-a)(1-a q)\left(1-a q^{2}\right) \cdots$.

Received: February 2, 2013

(c) 2013 Academic Publications, Ltd.

$\S$ Correspondence author url: www.acadpubl.eu 
Following Ramanujan, we defined

$$
\begin{gathered}
\varphi(q):=f(q, q)=\sum_{n=-\infty}^{\infty} q^{n^{2}}=\frac{(-q ;-q)_{\infty}}{(q ;-q)_{\infty}}, \\
\psi(q):=f\left(q, q^{3}\right)=\sum_{n=0}^{\infty} q^{\frac{n(n+1)}{2}}=\frac{\left(q^{2} ; q^{2}\right)_{\infty}}{\left(q ; q^{2}\right)_{\infty}} \\
f(-q):=f\left(-q,-q^{2}\right)=\sum_{n=-\infty}^{\infty}(-1)^{n} q^{\frac{n(3 n-1)}{2}}=(q ; q)_{\infty}
\end{gathered}
$$

and

$$
\chi(q):=\left(-q ; q^{2}\right)_{\infty} .
$$

On page 338 in his first notebook [11, p.338], Ramanujan defines

$$
a_{m, n}=\frac{n e^{\frac{-(n-1) \pi}{4} \sqrt{\frac{m}{n}}} \psi^{2}\left(e^{-\pi^{\sqrt{ }} \overline{m n}}\right) \varphi^{2}\left(-e^{-2 \pi^{\sqrt{ }} \overline{m n}}\right)}{\psi^{2}\left(e^{-\pi \sqrt{\frac{m}{n}}}\right) \varphi^{2}\left(-e^{-2 \pi \sqrt{\frac{m}{n}}}\right)} .
$$

He then, on pages 338 and 339, offers a list of eighteen particular values. All these eighteen values have been established by Berndt, Chan and Zhang [1]. An account of these can be found in Berndt's book [3], M. S. Mahadeva Naika and B. N. Dharmendra [4], also established some general theorems for explicit evaluations of the product of $a_{m, n}$ and found some new explicit values therefrom. Further results on $a_{m, n}$ can be found by Mahadeva Naika, Dharmendra and K. Shivashankar [6], and Mahadeva Naika and M. C. Mahesh Kumar [7].

In [8], Mahadeva Naika et al. defined the product

$$
b_{m, n}=\frac{n e^{\frac{-(n-1) \pi}{4} \sqrt{\frac{m}{n}}} \psi^{2}\left(-e^{-\pi^{\sqrt{ }} \overline{m n}}\right) \varphi^{2}\left(-e^{-2 \pi^{\sqrt{ }} \overline{m n}}\right)}{\psi^{2}\left(-e^{-\pi \sqrt{\frac{m}{n}}}\right) \varphi^{2}\left(-e^{-2 \pi \sqrt{\frac{m}{n}}}\right)} .
$$

They established general theorems for explicit evaluation of $b_{m, n}$ and obtained some particular values. Mahadeva Naika et al. [9] established general formulas for explicit values of Ramanujan's cubic continued fraction $V(q)$ in terms of the products $a_{m, n}$ and $b_{m, n}$ defined above, where

$$
V(q):=\frac{q^{1 / 3}}{1}+\frac{q+q^{2}}{1}+\frac{q^{2}+q^{4}}{1}+\frac{q^{3}+q^{6}}{1}+\cdots,|q|<1,
$$

and found some particular values of $V(q)$ 
In [10], Nipen Saikia defined the product of theta-fuctions $I_{m, n}$ as

$$
I_{m, n}=\frac{\psi(-q) \varphi\left(q^{m}\right)}{q^{(m-1) / 8} \psi\left(-q^{m}\right) \varphi(q)} ; \quad e^{-\pi \sqrt{\frac{n}{m}}}
$$

where $m$ and $n$ are positive real numbers. We establish several properties of the product $I_{m, n}$. They prove general formulas for explicit evaluations of evaluation of $I_{m, n}$ and find its explicit values.

In this paper, we establish several General Theorems and explicit evaluation of $I_{5, n}$.

Now we define a modular equation in brief. The ordinary hypergeometric series ${ }_{2} F_{1}(a, b ; c ; x)$ is defined by

$$
{ }_{2} F_{1}(a, b ; c ; x):=\sum_{n=0}^{\infty} \frac{(a)_{n}(b)_{n}}{(c)_{n} n !} x^{n}
$$

where $(a)_{0}=1,(a)_{n}=a(a+1)(a+2) \cdots(a+n-1)$ for any positive integer $n$, and $|x|<1$.

Let

$$
z:=z(x):={ }_{2} F_{1}\left(\frac{1}{2}, \frac{1}{2} ; 1 ; x\right)
$$

and

$$
q:=q(x):=\exp \left(-\pi \frac{{ }_{2} F_{1}\left(\frac{1}{2}, \frac{1}{2} ; 1 ; 1-x\right)}{{ }_{2} F_{1}\left(\frac{1}{2}, \frac{1}{2} ; 1 ; x\right)}\right),
$$

where $0<x<1$.

Let $r$ denote a fixed natural number and assume that the following relation holds:

$$
r \frac{{ }_{2} F_{1}\left(\frac{1}{2}, \frac{1}{2} ; 1 ; 1-\alpha\right)}{{ }_{2} F_{1}\left(\frac{1}{2}, \frac{1}{2} ; 1 ; \alpha\right)}=\frac{{ }_{2} F_{1}\left(\frac{1}{2}, \frac{1}{2} ; 1 ; 1-\beta\right)}{{ }_{2} F_{1}\left(\frac{1}{2}, \frac{1}{2} ; 1 ; \beta\right)} .
$$

Then a modular equation of degree $r$ in the classical theory is a relation between $\alpha$ and $\beta$ induced by (12). We often say that $\beta$ is of degree $r$ over $\alpha$ and $m:=\frac{z(\alpha)}{z(\beta)}$ is called the multiplier. We also use the notations $z_{1}:=z(\alpha)$ and $z_{r}:=z(\beta)$ to indicate that $\beta$ has degree $r$ over $\alpha$. 


\section{Preliminary Results}

Lemma 1. If $P=\frac{\psi(-q)}{q^{\frac{1}{2}} \psi\left(-q^{5}\right)}$ and $Q=\frac{\varphi(q)}{\varphi\left(q^{5}\right)}$, then

$$
P^{2}+P^{2} Q^{2}=5+Q^{2}
$$

Lemma 2. [5] If $P:=\frac{\varphi(q)}{\varphi\left(q^{5}\right)}$ and $Q:=\frac{\varphi\left(q^{2}\right)}{\varphi\left(q^{10}\right)}$, then

$$
\begin{aligned}
& \left(\frac{P}{Q}\right)^{2}+\left(\frac{Q}{P}\right)^{2}+(P Q)^{2}+\left(\frac{5}{P Q}\right)^{2}+16\left(\frac{P}{Q}-\frac{Q}{P}\right) \\
& =2\left(P^{2}+\frac{5}{P^{2}}\right)+2\left(Q^{2}+\frac{5}{Q^{2}}\right)+4 .
\end{aligned}
$$
then

Lemma 3. [3, Ch. 25, Entry 66, p.233] If $P=\frac{\varphi(q)}{\varphi\left(q^{5}\right)} \quad$ and $\quad Q=\frac{\varphi\left(q^{3}\right)}{\varphi\left(q^{15}\right)}$,

$$
P Q+\frac{5}{P Q}=-\left(\frac{P}{Q}\right)^{2}+\left(\frac{Q}{P}\right)^{2}+3\left(\frac{P}{Q}+\frac{Q}{P}\right) .
$$

Lemma 4. [5] If $P:=\frac{\varphi(q) \varphi\left(q^{4}\right)}{\varphi\left(q^{5}\right) \varphi\left(q^{20}\right)}$ and $Q:=\frac{\varphi(q) \varphi\left(q^{20}\right)}{\varphi\left(q^{5}\right) \varphi\left(q^{4}\right)}$, then

$$
\begin{aligned}
& Q^{4}+\frac{1}{Q^{4}}-112\left(Q^{3}+\frac{1}{Q^{3}}\right)+1440\left(Q^{2}+\frac{1}{Q^{2}}\right) \\
& -3184\left(Q+\frac{1}{Q}\right)+7316=8\left(P+\frac{1}{P}\right)\left[22\left(Q^{2}+\frac{1}{Q^{2}}\right)\right. \\
& \left.-31\left(Q+\frac{1}{Q}\right)+170\right]-2\left(P^{2}+\frac{5^{2}}{P^{2}}\right)\left[3\left(Q^{2}+\frac{1}{Q^{2}}\right)\right. \\
& \left.+24\left(Q+\frac{1}{Q}\right)+64\right]+4\left(P^{3}+\frac{5^{3}}{P^{3}}\right)\left[\left(Q+\frac{1}{Q}\right)+4\right] .
\end{aligned}
$$

Lemma 5. [5] If $P:=\frac{\varphi(q)}{\varphi\left(q^{5}\right)}$ and $Q:=\frac{\varphi\left(q^{5}\right)}{\varphi\left(q^{25}\right)}$, then

$$
\begin{aligned}
& \frac{Q^{3}}{P^{3}}-\frac{5 Q^{2}}{P^{2}}-\frac{15 Q}{P}+5\left(P Q+\frac{5}{P Q}\right)+5\left(Q^{2}+\frac{5}{P^{2}}\right) \\
& =P^{2} Q^{2}+\frac{5^{2}}{P^{2} Q^{2}}+15 .
\end{aligned}
$$


Lemma 6. [10]

$$
I_{m, 1}=1
$$

Lemma 7. [10]

$$
I_{m, n} I_{m, 1 / n}=1
$$

\section{General Theorems and Explicit Evaluations of $I_{5, n}$}

Theorem 8. If $X:=I_{5, n}$ and $Y:=I_{5,4 n}$ then

$$
\begin{aligned}
& \left(41-19080 y^{6}-477576 y^{10}+5832 y^{14}+59049 y^{16}-3588 y^{4}\right. \\
& \left.+456 y^{2}+190134 y^{8}+378108 y^{12}\right) x^{16}+\left(809280 y^{14}+5832 y^{16}\right. \\
& -1621792 y^{4}-10888000 y^{6}-1394976 y^{12}-63680 y^{2}+456 \\
& \left.-7002432 y^{10}-15707216 y^{8}\right) x^{14}+\left(378108 y^{16}+12465808 y^{12}\right. \\
& -29385584 y^{4}-135695328 y^{6}-3588-12611040 y^{10}-1621792 y^{2} \\
& \left.-15502360 y^{8}-1394976 y^{14}\right) x^{12}+\left(295520720 y^{8}-7002432 y^{14}\right. \\
& -12611040 y^{12}-29291200 y^{10}-477576 y^{16}-19080 \\
& \left.-315184320 y^{6}-10888000 y^{2}-135695328 y^{4}\right) x^{10}+\left(295520720 y^{6}\right. \\
& -15502360 y^{12}+190134 y^{16}+295520720 y^{10}-15707216 y^{2} \\
& \left.+190134-15707216 y^{14}+1364493764 y^{8}-15502360 y^{4}\right) x^{8} \\
& +\left(295520720 y^{8}-315184320 y^{10}-29291200 y^{6}-7002432 y^{2}\right. \\
& \left.-135695328 y^{12}-477576-19080 y^{16}-12611040 y^{4}-10888000 y^{14}\right) x^{6} \\
& +\left(378108-15502360 y^{8}-1394976 y^{2}-29385584 y^{12}+12465808 y^{4}\right. \\
& \left.-12611040 y^{6}-135695328 y^{10}-1621792 y^{14}-3588 y^{16}\right) x^{4} \\
& +\left(456 y^{16}-15707216 y^{8}+809280 y^{2}-10888000 y^{10}+5832\right. \\
& \left.-63680 y^{14}-1621792 y^{12}-7002432 y^{6}-1394976 y^{4}\right) x^{2} \\
& +59049-19080 y^{10}+5832 y^{2}+456 y^{14}-477576 y^{6} \\
& +41 y^{16}+378108 y^{4}+190134 y^{8}-3588 y^{12}=0 .
\end{aligned}
$$

Proof. Employing the definition of $I_{m, n}(9)$ with $m=5$, we obtain

$$
I_{5, n}=\frac{\psi(-q) \varphi\left(q^{5}\right)}{q^{(1 / 2} \psi\left(-q^{5}\right) \varphi(q)} ; \quad e^{-\pi \sqrt{\frac{n}{5}}}
$$


By using lemma (1), we obtain

$$
P=\sqrt{\frac{Q^{2}+5}{Q^{2}+1}} .
$$

The above two equations (21) and (22) can be written as

$$
I_{5, n}=\frac{Q^{2}+5}{Q^{4}+Q^{2}},
$$

where $Q=\frac{\varphi(q)}{\varphi\left(q^{5}\right)}$, the above the equation (23) can be written as

$$
Q^{2}=\frac{(1-a)+\sqrt{a^{2}+18 a+1}}{2 a},
$$

where $a=I_{5, n}$.

Employing the equation (24) in (14), we obtain (8)

Corollary 9. We have

$$
\begin{gathered}
I_{5,2}=\left[\frac{(19-8 \sqrt{5})(29+20 \sqrt{2})}{41}\right]^{1 / 2}, \\
I_{5,1 / 2}=\left[\frac{(19+8 \sqrt{5})(29-20 \sqrt{2})}{41}\right]^{1 / 2} \cdot \\
I_{5,4}=\left[\frac{7711(17511+8280 \sqrt{5}-106 d+4 \sqrt{38555} k)}{4017413}\right]^{1 / 2}, \\
I_{5,1 / 4}=\left[\frac{7711(17511+8280 \sqrt{5}-106 d-4 \sqrt{38555} k)}{4017413}\right]^{1 / 2} .
\end{gathered}
$$

where, $k:=\sqrt{(5837+2760 \sqrt{5})(10193698+4580131 \sqrt{5}-92532 d)}$, $d:=\sqrt{24371+10909 \sqrt{5}}$

Proof. Setting $n=1 / 2$ in Theorem (8) and using the Lemma (7), we obtain $\left(41-2204 I_{5,2}+4726 I_{5,2}^{2}-2204 I_{5,2}^{3}+41 I_{5,2}^{4}\right)\left(I_{5,2}+1\right)^{4}\left(I_{5,2}^{2}+18 I_{5,2}+1\right)^{4}=0$ 
Since the root of the second and third factors are imaginary and $I_{5,2}<10$ we deduce that

$$
41-2204 I_{5,2}+4726 I_{5,2}^{2}-2204 I_{5,2}^{3}+41 I_{5,2}^{4}=0
$$

On solving the above equation (29), we arrive at the equations (25) and (26).

Setting $n=1$ in Theorem (8) and using the Lemma (6), we obtain

$$
\begin{aligned}
& \left(521-140088 I_{5,4}^{2}-716292 I_{5,4}^{4}-842376 I_{5,4}^{6}+7396470 I_{5,4}^{8}-842376 I_{5,4}^{10}\right. \\
& \left.-716292 I_{5,4}^{12}-140088 I_{5,4}^{14}+521 I_{5,4}^{16}\right)\left(59 I_{5,4}^{8}+6284 I_{5,4}^{6}-8286 I_{5,4}^{4}\right. \\
& \left.+6284 I_{5,4}^{2}+59\right)^{2}\left(I_{5,4}^{16}-88 I_{5,4}^{14}+8668 I_{5,4}^{12}-38248 I_{5,4}^{10}+102598 I_{5,4}^{8}\right. \\
& \left.-38248 I_{5,4}^{6}+8668 I_{5,4}^{4}-88 I_{5,4}^{2}+1\right)^{2}=0
\end{aligned}
$$

Since the root of the second and third factors are imaginary and $I_{5,4}>0$ we deduce that

$$
\begin{aligned}
& \left(521-140088 I_{5,4}^{2}-716292 I_{5,4}^{4}-842376 I_{5,4}^{6}+7396470 I_{5,4}^{8}\right. \\
& \left.-842376 I_{5,4}^{10}-716292 I_{5,4}^{12}-140088 I_{5,4}^{14}+521 I_{5,4}^{16}\right)=0
\end{aligned}
$$

by above equation (31) can be written as

$$
521 z^{4}-140088 z^{3}-718376 z^{2}-422112 z+8830096=0, z=I_{5,4}^{2}+I_{5,4}^{-2} .
$$

On solving the above equation (32), we arrive at the equations (27).

Theorem 10. If $X:=I_{5, n}$ and $Y:=I_{5,9 n}$ then

$$
\begin{aligned}
& \left(4908 y^{2}+209+2940 y^{6}+9 y^{8}-7362 y^{4}\right) x^{16}+\left(4908+189552 y^{8}\right. \\
& \left.-317359 y^{6}+144339 y^{2}+35670 y^{4}-9 y^{14}-2994 y^{12}-9819 y^{10}\right) x^{14} \\
& +\left(35670 y^{2}-2994 y^{14}+5149482 y^{10}-944110 y^{12}+8930322 y^{6}\right. \\
& \left.-7362-3476010 y^{4}-10022406 y^{8}\right) x^{12}+\left(2940+43492872 y^{8}\right. \\
& +8930322 y^{4}-317359 y^{2}-25684089 y^{10}-30624573 y^{6} \\
& \left.+5149482 y^{12}-9819 y^{14}\right) x^{10}+\left(43492872 y^{6}-68614774 y^{8}\right. \\
& -10022406 y^{4}+189552 y^{2}+9-10022406 y^{12}+43492872 y^{10} \\
& \left.+189552 y^{14}+9 y^{16}\right) x^{8}+\left(2940 y^{16}-317359 y^{14}-9819 y^{2}\right. \\
& +43492872 y^{8}-25684089 y^{6}-30624573 y^{10}+5149482 y^{4} \\
& \left.+8930322 y^{12}\right) x^{6}+\left(-7362 y^{16}-3476010 y^{12}-10022406 y^{8}\right. \\
& \left.-944110 y^{4}+35670 y^{14}-2994 y^{2}+8930322 y^{10}+5149482 y^{6}\right) x^{4}
\end{aligned}
$$




$$
\begin{aligned}
& +\left(35670 y^{12}+144339 y^{14}-2994 y^{4}+189552 y^{8}+4908 y^{16}\right. \\
& \left.-9819 y^{6}-317359 y^{10}-9 y^{2}\right) x^{2}-7362 y^{12}+4908 y^{14}+9 y^{8} \\
& +209 y^{16}+2940 y^{10}=0
\end{aligned}
$$

Proof. Employing the equation (24) in (15), we obtain (33)

Corollary 11. We have

$$
\begin{aligned}
& I_{5,3}=\frac{(7+\sqrt{5}) \sqrt{11}}{22} \\
& I_{5,1 / 3}=\frac{(7-\sqrt{5}) \sqrt{11}}{22} . \\
& I_{5,9}=\left[\frac{(15-8 \sqrt{3}) 6 \sqrt{5}+23(8-3 \sqrt{5})}{41}\right]^{1 / 2} . \\
& I_{5,1 / 9}=\left[\frac{(15+8 \sqrt{3}) 6 \sqrt{5}-23(8+3 \sqrt{5})}{41}\right]^{1 / 2} .
\end{aligned}
$$

Proof. Employing Theorem (10), Lemma (7) and (6), solving the resulting equation for $I_{5,3}, I_{5,9}$ and nothing that $I_{5,3}<1$ and $I_{5,9}<1$, we arrive (34)(37)

Theorem 12. If $X:=I_{5, n}$ and $Y:=I_{5,25 n}$ then

$$
\begin{aligned}
& \left(5-330 y^{4}+840 y^{2}+420 y^{6}-291 y^{8}+60 y^{10}\right) x^{20}+\left(-376735 y^{10}\right. \\
& -60 y^{20}+840+132000 y^{12}-384091 y^{6}-309 y^{18}+6390 y^{16} \\
& \left.-31185 y^{14}+803920 y^{4}+152720 y^{2}+481790 y^{8}\right) x^{18}+\left(803920 y^{2}\right. \\
& -29291516 y^{4}+130285890 y^{10}-931795 y^{20}+63203590 y^{6} \\
& -117602260 y^{8}+67420470 y^{14}-33495204 y^{16}-97728055 y^{12} \\
& \left.-330+8886970 y^{18}\right) x^{16}+\left(63203590 y^{4}+420+11199050 y^{16}\right. \\
& +3713550 y^{12}-1530065 y^{18}+184185180 y^{10}-384091 y^{2} \\
& \left.+62340 y^{20}+88653490 y^{6}-228679750 y^{8}-35882274 y^{14}\right) x^{14} \\
& +\left(9481830 y^{14}-46268690 y^{10}+74820 y^{18}-21431094 y^{12}\right. \\
& +481790 y^{2}-117602260 y^{4}-291+234609170 y^{8}-8730 y^{20} \\
& \left.-228679750 y^{6}-1223835 y^{16}\right) x^{12}+\left(60-340650 y^{16}+11025 y^{18}\right. \\
& -376735 y^{2}+4320 y^{20}+130285890 y^{4}+1274110 y^{12}-3446698 y^{10}
\end{aligned}
$$




$$
\begin{aligned}
& \left.+184185180 y^{6}-46268690 y^{8}+681020 y^{14}\right) x^{10}+\left(132000 y^{2}\right. \\
& +132000 y^{2}-771150 y^{14}-21431094 y^{8}-891 y^{20}-270 y^{18} \\
& +103410 y^{16}+3713550 y^{6}-97728055 y^{4}+1274110 y^{10} \\
& \left.+2458150 y^{12}\right) x^{8}+\left(681020 y^{10}-891 y^{18}+103410 y^{14}\right. \\
& +9481830 y^{8}-31185 y^{2}+67420470 y^{4}-35882274 y^{6} \\
& \left.-270 y^{16}-771150 y^{12}\right) x^{6}+\left(103410 y^{12}-340650 y^{10}\right. \\
& -891 y^{16}-33495204 y^{4}+6390 y^{2}+11199050 y^{6}-1223835 y^{8} \\
& \left.-270 y^{14}\right) x^{4}+\left(74820 y^{8}-1530065 y^{6}+8886970 y^{4}+11025 y^{10}\right. \\
& \left.-891 y^{14}-891 y^{12}-309 y^{2}-270 y^{12}\right) x^{2}-931795 y^{4}+4320 y^{10} \\
& -8730 y^{8}+62340 y^{6}-60 y^{2}=0 .
\end{aligned}
$$

Proof. Employing the equation (24) in (17), we obtain (38)

Corollary 13. We have

$$
\begin{aligned}
& I_{5,5}=\sqrt{\frac{13+6 \sqrt{5}}{11}}, \\
& I_{5,1 / 5}=\sqrt{6 \sqrt{5}-13} .
\end{aligned}
$$

Proof. Employing Theorem (12) and Lemma (7), solving the resulting equation for $I_{5,3}$ and nothing that $I_{5,3}<1$, we arrive (39) and (40)

\section{References}

[1] B.C. Berndt, H.H. Chan, L.-C. Zhang, Ramanujan's remarkable product of the theta-function, Proc. Edinburgh Math. Soc., 40 (1997), 583-612.

[2] B.C. Berndt, Ramanujan's Notebooks, Part III, Springer-Verlag, New York (1991).

[3] B.C. Berndt, Ramanujan's Notebooks, Part V, Springer-Verlag, New York (1998).

[4] M.S. Mahadeva Naika, B.N. Dharmendra, On some new general theorems for the explicit evaluations of Ramanujan's remarkable product of thetafunction, Ramanujan J., 15, No. 3 (2008), 349-366. 
[5] M.S. Mahadeva Naika, B.N. Dharmendra, S. Chandankumar, On some new modular relations for Ramanujan's parameter $\mu(q)$ and their evaluations, IJPAM, Accepted.

[6] M.S. Mahadeva Naika, B.N. Dharmendra, K. Shivashankara, On some new explicit evaluations of Ramanujan's remarkable product of theta-function, South East Asian J. Math. Math. Sci., 5, No. 1 (2006), 107-119.

[7] M.S. Mahadeva Naika, M.C. Maheshkumar, Explicit evaluations of Ramanujan's remarkable product of theta-function, Adv. Stud. Contemp. Math., 13, No. 2 (2006), 235-254.

[8] M.S. Mahadeva Naika, M.C. Maheshkumar, K. Sushan Bairy, On some remarkable product of theta-function, Aust. J. Math. Anal. Appl., 5, No. 1 (2008), 1-15.

[9] M.S. Mahadeva Naika, M.C. Maheshkumar, K. Sushan Bairy, General formulas for explicit evaluations of Ramanujan's cubic continued fraction, Kyungpook Math. J., 49, No. 3 (2009), 435-450.

[10] Nipen Saikia, A product of theta-functions analogous to Ramanujan's remarkable product of theta-functions and applications, Hindawi Publishing Corporation, Journal of Mathematics, Volume 2013.

[11] S. Ramanujan, Notebooks (2 volumes), Bombay, Tata Institute of Fundamental Research (1957). 